\title{
Fármacos antimicrobianos e antivirais com potencial uso terapêutico para a COVID-19
}

\author{
Antimicrobial and antiviral agents with potential therapeutic use \\ for COVID-19
}

Recebido em: 12/05/2020 Aceito em: $17 / 06 / 2020$
Hellen Cryslen Bernardo BEZERRA; Daniel Parente XAVIER; Tatiana Paschoalette Rodrigues BACHUR; Gislei Frota ARAGÃO Universidade Estadual do Ceará, CCS, Curso de Medicina. Av. Dr. Silas Munguba, 1700, Itaperi, CEP: 60.714-903. Fortaleza, CE, Brasil.

E-mail:tatiana.bachur@uece.br

\section{ABSTRACT}

The disease caused by the new SARS-CoV-2 coronavirus, called COVID-19, has already infected more than 6.5 million people worldwide. To date, there is no proven effective treatment against the virus, but some drugs already used in other diseases have shown effectiveness in vitro or even in humans, such as some antimicrobial agents. The objective of this bibliographic research was to provide an overview of the published evidence, up to the research date, about experimental studies and clinical trials that used antimicrobial drugs as a treatment proposal for the disease by the new coronavirus. 75 articles from journals indexed in the MEDLINE database were included. The results showed that the drugs Lopinavir/Ritonavir, chloroquine and hydroxychloroquine demonstrated to act in the clinical improvement of patients diagnosed with COVID-19. However, more evidence is needed to confirm the data currently available.

Keywords: Coronavirus infections; Drug therapy; Anti-infective agents.

\section{RESUMO}

A doença causada pelo novo coronavírus SARS-CoV-2, denominada COVID-19, já infectou mais de 6,5 milhões de pessoas no mundo. Até o presente momento, não há tratamento comprovadamente eficaz contra o vírus, mas alguns medicamentos já utilizados em outras doenças demonstraram eficácia in vitro ou mesmo em humanos, como alguns agentes antimicrobianos. O objetivo desta pesquisa bibliográfica foi fornecer uma visão geral das evidências publicadas, até a data de pesquisa, acerca dos estudos experimentais e ensaios clínicos que utilizaram fármacos antimicrobianos como proposta de tratamento para a doença pelo novo coronavírus. Foram incluídos 75 artigos de periódicos indexados na base de dados MEDLINE. Os resultados mostraram que os fármacos Lopinavir/Ritonavir, cloroquina e hidroxicloroquina demonstraram atuar na melhora clínica de pacientes diagnosticados com COVID-19. No entanto, mais evidências são necessárias para a confirmação dos dados disponíveis atualmente.

Palavras-chave: Infecções por coronavírus; Tratamento farmacológico; Anti-infecciosos. 


\section{INTRODUÇÃO}

No final de 2019, foi relatado um surto de uma nova pneumonia de causa desconhecida na cidade de Wuhan, província de Hubei, na China. Em janeiro de 2020, a análise de amostras de lavado broncoalveolar coletadas em três pacientes com o quadro pneumonia grave no Hospital Wuhan Jinyintan, possibilitou a identificação do agente etiológico: um vírus, nomeado SARS-CoV-2, com características morfológicas consistentes com a da família Coronaviridae (1).

Desde então, a doença por SARS-CoV-2, denominada COVID-19 pela Organização Mundial da Saúde (OMS), apresentou uma rápida disseminação geográfica em um curto período de tempo, associada a uma alta taxa de contaminação, sendo decretado o estado de pandemia no dia 11 de março de 2020. Atualmente, já foram registrados mais de 6,5 milhões de casos confirmados e mais de 390 mil mortes no mundo (2).

Até o momento, sabe-se que o SARS-CoV-2, provavelmente, usa o receptor ACE2 para entrar nas células humanas (3). No início da doença, os sintomas mais comuns são febre, tosse e mialgia, evoluindo para pneumonia e, quando grave, para maiores complicações, como a síndrome do desconforto respiratório agudo grave (SDRA) (4).

Ainda não há tratamentos ou medicamentos específicos anti-SARS-CoV-2. Na corrida contra a COVID-19, a principal medida terapêutica encontrada tem sido o uso de medicamentos já existentes para outras doenças e disponíveis na clínica, pelo menos a curto prazo. Dessa forma, o objetivo do presente trabalho foi de investigar os atuais estudos experimentais e ensaios clínicos que tem fármacos antimicrobianos como proposta de tratamento para a doença pelo novo coronavírus, buscando fornecer uma visão geral das evidências publicadas até a data de pesquisa.

\section{MÉTODO}

Utilizando os periódicos indexados na base de dados MEDLINE, foi conduzida uma revisão de literatura para identificar artigos com foco em fármacos antivirais e antimicrobianos utilizados no tratamento da COVID-19. Para a busca da literatura pertinente foram utilizados os seguintes descritores MeSH (Medical Subject Headings) e suas combinações: "anti-infective agents", "antiviral agents", "antiparasitic agents", "antibacterial agents", "COVID-19 drug treatment", sars-cov-2 e COVID-19. Foram incluídos 75 artigos considerados relevantes para o trabalho publicados entre 1983 até maio de 2020. Artigos relevantes adicionais foram identificados a partir da revisão das citações referenciadas. Os ensaios clínicos foram identificados por um levantamento no site ClinicalTrials.Gov.

\section{RESULTADOS E DISCUSSÃO}

Os principais agentes encontrados como potenciais fármacos contra a COVID-19 são descritos a seguir.

\section{AGENTES ANTIVIRAIS}

\section{Remdesivir}

O Remdesivir é um análogo da adenosina desenvolvido para o tratamento da infecção causada pelo vírus Ebola (5). O fármaco tem como alvo a enzima RNA polimerase RNA dependente (RpRd), atuando como inibidor competitivo em relação ao ATP e interferindo nas reações de metilação da S-adenosilmetionina (SAM) por meio da inibição da hidrolase da S-adenosil-homocisteína (SAH) (5).

Esse fármaco se mostrou eficaz na inibição do SARS-CoV-2, MERS-CoV e SARS-CoV-1 em testes realizados in vitro, inclusive em associação com outros fármacos, como Cloroquina e IFN-beta $(6,7)$.

Atualmente, o Remdesivir encontra-se em fase III de ensaio clínico conduzido pelo fabricante (GileadSciences, Inc.) $(8,9)$. Entretanto, um estudo realizado com 236 pacientes em dez hospitais da cidade de Wuhan, China, demonstrou que o Remdesivir possui baixa efetividade no tratamento da COVID-19 (10). 
Os efeitos colaterais são de características gastrointestinais (náuseas e vômitos), além de reações alérgicas e elevação de transaminases (11).

\section{Favipiravir}

O Favipiravir, também conhecido como Avigan, T-705 ou Favilavir, é um antiviral, derivado da pirazinamida, utilizado no tratamento de várias doenças causadas por vírus de RNA, como febre amarela, e infecções causadas por flavivírus, arenavírus, vírus do Nilo ocidental (12). Além disso, foi usado, experimentalmente, contra o vírus Ebola (13). É aprovado no Japão para tratamento da gripe e, em fevereiro de 2020, passou a ser testado no tratamento da COVID-19 na China $(14,15)$.

É um pró-fármaco cujo mecanismo de ação ocorre no interior da célula quando é ribosilado e fosforilado formando o metabólito ativo favipiravir ibofuranosil-5'-trifosfato (T-705-RTP) (16). O T-705-RTP compete com os nucleosídeos de purina interferindo na replicação viral por incorporação ao RNA do vírus, assim sendo, inibirá a RNA-polimerase dependente de RNA (RdRp) (17). Atualmente, o fármaco encontra-se em fase três de ensaios clíni$\cos$ (18). Em um estudo off-label foi demonstrado a eficácia de Favipiravir no tratamento de pacientes com COVID-19 (19). Ademais, o medicamento também se mostrou eficaz em estudos in vitro contra o novo coronavírus (20).

O Favipiravir pode causar efeitos teratogênicos, além de já ter sido observado o prolongamento do intervalo QTc, entre outras arritmias cardíacas, em paciente infectado pelo vírus Ebola, o que deve ser levado em conta antes de ser utilizado em pacientes apresentando cardiopatias (21).

\section{Lopinavir/Ritonavir}

Lopinavir (LPV) é um antirretroviral utilizado no tratamento do HIV (Vírus da Imunodeficiência Humana) (22). É um potente inibidor da protease do ácido aspártico. O Lopinavir é administrado em combinação com o Ritonavir (RTV), associação que é capaz de potencializar a atividade farmacocinética do primeiro, devido à inibição do citocromo P-450 CIP3A, com consequente aumento do tempo de meia-vida do LPV (23).
As proteases são fundamentais na formação do vírus; assim, sua inibição impede a multiplicação viral e a destruição da célula do hospedeiro infectado (24).

A associação Lopinavir/Ritonavir (LPV/RTV) tem mostrado alguma eficácia em pacientes infectados com SARS-CoV-1, bem como ação in vitro contra o SARS-CoV-2 $(25,26)$. Contudo, LPV foi desenvolvido para atuar na estrutura da protease do ácido aspártico do HIV, que é estruturalmente diferente da protease do SARS-CoV-2 (27).

Um estudo realizado no St. Michael's Hospital recrutou 1220 pacientes infectados com SARS-CoV-2 para testar a associação LPV/RTV e, atualmente, se encontra em fase III (28). Um ensaio clínico realizado em Guangzhou 8th People’s Hospital, com 125 pacientes infectados com SARS-CoV-2, se apresenta em fase IV (29). Um ensaio clínico realizado com 199 pacientes, dos quais 99 receberam tratamento por 14 dias com LPV/RTV e outros 100 receberam o tratamento padrão (oxigênio, antibióticos e suporte vasopressor) não mostrou melhoras clínicas dos pacientes tratados com LPV/RTV (30), enquanto outros trabalhos relataram a eficiência da combinação LPV/RTV no combate a COVID-19 $(31,32)$.

Os efeitos adversos mais comumente observados em pacientes que utilizam LPV/RTV são distúrbios gastrointestinais, como náuseas, vômitos e diarreia (30). Também podem ser observadas, em uso off-label, arritmias cardíacas como o prolongamento do intervalo QTc (33).

\section{Oseltamivir}

O Oseltamivir é um inibidor ativo da neuraminidase (34). Trata-se de um fármaco utilizado no tratamento de infecções causadas pelo ortomixovírus incluindo, também, as influenzas do tipo A e B e o Vírus Sincicial Respiratório (35). A ação do Oseltamivir sobre as neuraminidases, glicoproteínas de liberação dos viríons, impede a saída do vírus da célula infectada para outra célula inibindo a progressão da infecção (36). O Oseltamivir é um pró-fármaco administrado por via oral, sendo bioativado no fígado (36).

A associação do Oseltamivir com outros fármacos para tratamento da COVID-19 tem sido tes- 
tada (37). O Rajavithi Hospital combinou Oseltamivir, Favipiravir e Cloroquina no tratamento de 80 pacientes infectados com SARS-CoV-2, e o estudo se encontra em fase III (38).

Outro estudo realizado na cidade de Wuhan, na China, com 138 pacientes infectados com SARS-CoV-2, dos quais 124 (89,9\%), em diferentes fases da doença e com diferentes complicações clínicas, receberam tratamento com Oseltamivir, glicocorticoides, azitromicina e terapia antibacteriana. Desses, 4,3\% dos pacientes foram a óbito (39).

O principal efeito adverso do Oseltamivir são os desconfortos gastrointestinais e náuseas, que podem ser diminuídos pela ingestão do medicamento juntamente com alimento (40).

\section{Darunavir}

O Darunavir é um inibidor de protease de segunda geração aprovado para o tratamento inicial de pacientes com HIV, bem como para aqueles que possuam a doença há mais tempo e que resistem a outros inibidores de proteases (41). O Darunavir deve ser administrado com alimentação para aumentar a sua absorção e é biotransformado intensamente pelas CIP3A4 (42).

Trata-se, portanto, de inibidor reversível da aspartil protease do HIV, enzima responsável pela clivagem de um poliproteína viral que é responsável pela fabricação das enzimas transcriptase reversa, proteases e integrases e algumas outras proteínas que formam estruturalmente o vírus (43).

A efetividade do Darunavir contra o SARS-CoV-2 vem sendo investigada (44). O uso off-label do Darunavir na COVID-19 foi aprovado nos Estados Unidos (45). Experiências com células indicaram que o Darunavir inibiu a replicação do SARS-CoV-2 in vitro e que sua eficiência foi de 280 vezes maior do que no grupo de células não tratadas (46). Ensaios clínicos utilizando o Darunavir e Cobacistat estão em andamento, em fase III, para verificar a eficácia e segurança do fármaco em pacientes com COVID-19 (47).

Os efeitos adversos mais observados na prática clínica no tratamento medicamentoso com Darunavir são: náuseas, desconforto abdominal de duração variada e distúrbios lipídicos, efeitos co- muns a outros fármacos inibidores de protease do $\operatorname{HIV}(48,49)$.

\section{Umifenovir}

Umifenovir (Arbidol®) é um antiviral utilizado na Rússia e na China para tratamento da influenza, não sendo aprovado pelo FDA, Estados Unidos, para este fim (50). Assim, o Umifenovir é conhecido, sobretudo, nos países em que seu uso é aprovado para o tratamento da influenza e também da hepatite C (51).

Esse fármaco atua impedindo a fusão da membrana do vírus à membrana plasmática da célula (50). No vírus da influenza, por exemplo, o Umifenovir liga-se à hemaglutinina (HA), glicoproteína que tem função de ligar o vírus à célula, causando uma instabilidade na HA, impedindo a fusão do vírus com a membrana celular e, consequentemente, sua entrada na célula, culminando na inibição da infecção (52).

Um estudo tem procurado demonstrar a eficácia do Umifenovir no combate ao SARS-CoV-2 (53). Um ensaio realizado com 50 pacientes, dos quais 34 receberam tratamento com Liponavir/ Ritonavir (LPV/RTV) e 16 receberam tratamento com Umifenovir, mostrou que após 14 dias da admissão, os pacientes tratados com Umifenovir tiveram a carga viral zerada, enquanto $44,1 \%$ pacientes que receberam a combinação LPV/RTV ainda apresentavam carga de SARS-CoV-2 (54).

Outro ensaio clínico analisou um grupo de 33 pacientes, todos maiores de 18 anos e com infecção por SARS-CoV-2. Foram separados dois grupos: 16 pacientes receberam a combinação de LPV/ RTV com Umifenovir enquanto 17 pacientes receberam apenas LPV/RTV. Ao fim de sete dias foi avaliada a evolução da pneumonia e a tomografia computadorizada de tórax dos dois grupos. O grupo ao qual foi administrada terapia LPV/RTV mais Umifenovir apresentou melhoras clínicas mais significativas do que o grupo tratado apenas com LPV/RTV (55).

Os principais efeitos adversos do Umifenovir incluem efeitos gastrointestinais, como diarreia, vômito e náuseas. Em alguns casos, podem ocorrer reações de hipersensibilidade (56). 


\section{ANTIPARASITÁRIOS E ANTIMICROBIANOS}

\section{Cloroquina}

A cloroquina (CLQ), uma 4-aminoquinolona, é uma base fraca utilizada há mais de 70 anos no tratamento da malária e de algumas doenças autoimunes. Quando administrada, a CLQ difunde-se passivamente pelas membranas celulares em um estado lipofílico desprotonado. No interior da célula, ao entrar em vesículas ácidas, como os lisossomos, a molécula é protonada e fica aprisionada no compartimento $(57,58)$. Consequentemente, o fármaco age promovendo um aumento do $\mathrm{pH}$ intravesicular por consumir os íons hidrogênio no local, inibindo várias enzimas que exercem sua função em valores baixos de $\mathrm{pH}(59,60)$.

Sua administração é feita como sal fosfato, o qual é absorvido no trato gastrointestinal, possui uma meia-vida longa e grande volume de distribuição corporal, devido à sua ampla deposição tecidual. A metabolização é feita pelas enzimas do CIP450 e sua excreção é renal, um alerta para sua aplicação em pacientes portadores de nefropatias (61).

Recentemente, estudos têm demonstrado a atividade in vitro da cloroquina contra uma gama de vírus, incluindo o SARS-CoV (62). Foi sugerido que esse efeito antiviral nos coronavírus pode ocorrer pela interferência da fusão do vírus com a célula, assim como a inibição de inúmeros outros processos fundamentais para o vírus, como a replicação de ácidos nucleicos e glicosilação de proteínas virais (63).

Sua atividade antiviral contra o SARS-CoV-2 ainda não foi totalmente elucidada, embora tenha sido relatado que a CLQ bloqueia a interação entre a proteína $\mathrm{S}$ do SARS-CoV-2 e gangliosídeos presentes no trato respiratório (64). Ademais, foi sugerido que a CLQ bloqueia o transporte de SARS-CoV-2 para os endolisosomos, o que seria uma etapa necessária para a liberação do genoma viral (65).

Um estudo in vitro demonstrou que a concentração de CLQ necessária para alcançar me- tade do seu efeito máximo $\left(\mathrm{EC}_{50}\right)$ no bloqueio da infecção por COVID-19 é de 1,13 $\mu \mathrm{M}$, um valor baixo e com possibilidade de ser clinicamente aplicável em pacientes infectados (6). No início de fevereiro, o Conselho do Estado da China anunciou que ensaios clínicos realizados na China, envolvendo mais de 100 pacientes, demonstraram que a CLQ tem eficácia superior ao tratamento controle da COVID-19, além de melhorar os achados de imagem pulmonar, sendo recomendada a sua inclusão nas Diretrizes para Prevenção, Diagnóstico e Tratamento da Pneumonia Causada pelo COVID-19, da Comissão Nacional de Saúde da República Popular da China (66). Até a data da presente pesquisa, 15 ensaios clínicos (EC) intervencionistas, entre as fases I e IV, estão analisando a eficácia da CLQ como uma terapia anti-COVID-19 em monoterapia (8 EC), com outros adjuvantes (6 EC) ou como agente profilático (1 EC), disponíveis no site ClinicalTrials.GOV.

A recomendação do Ministério da Saúde do Brasil para o seu uso é como adjuvante no tratamento das formas graves, sendo a posologia de 3 comprimidos de $150 \mathrm{mg}$ 2x/dia no primeiro dia, seguidos de 3 comprimidos de $150 \mathrm{mg} \mathrm{1x/dia} \mathrm{no}$ segundo, terceiro, quarto e quinto dias (67). Ressalta-se que, embora a CLQ tenha um perfil de segurança conhecido e bom, alguns efeitos colaterais graves podem ocorrer durante o tratamento e devem ser sempre monitorados, como retinopatias, prolongamento do intervalo QT e efeitos neuropsiquiátricos (68).

\section{Hidroxicloroquina}

A hidroxicloroquina (HCQ) é um medicamento antimalárico, mais utilizado após o surgimento de cepas de $P$. falciparum resistentes a CLQ, e antirreumático, bastante utilizado na artrite reumatoide e no lúpus eritematoso sistêmico. Sua estrutura química é parecida com a da CLQ, diferindo pela presença de um grupo hidroxila ao final de sua cadeia lateral (65). Por pertencer à mesma classe de fármaco que a CLQ, a farmacodinâmica e a farmacocinética de ambas são similares; no entanto, há mudanças nas suas indicações clínicas e doses terapêuticas (69). 
Para a atividade anti-SARS-CoV-2 in vitro, HQC apresentou uma concentração para atingir metade do efeito máximo $\left(\mathrm{EC}_{50}\right)$ no valor de 0,72 $\mu \mathrm{M}$, após $48 \mathrm{~h}$, apontando ser uma possível melhor e mais potente opção para o tratamento da infecção por COVID-19 em comparação com a CLQ (63). Um estudo não randomizado demonstrou que $70 \%$ de pacientes $(\mathrm{n}=22)$ tratados com $600 \mathrm{mg}$ de HCQ por dia tiveram cura no sexto dia de tratamento, comparado com $12,5 \%$ dos pacientes do grupo controle $(n=20)$. Entre os pacientes tratados com HCQ, seis receberam azitromicina (AZ) em combinação e 100\% desses pacientes apresentaram amostra nasofaríngea negativa no sexto dia (70). Além disso, entre 80 pacientes tratados com HCQ e AZ na França, 97,5\% apresentaram melhora clínica, permanecendo na UTI, em média, apenas cinco dias (71). No entanto, há resultados divergentes que relataram não haver benefícios clínicos da HCQ na terapia anti-COVID-19. Segundo Molina e cols (2020), ao usar o mesmo regime terapêutico relatado por Gautret e cols (2020) em 11 pacientes, sendo $73 \%$ deles portadores de comorbidades, os swabs nasofaríngeosainda eram positivos em 8 pacientes após 5 dias do início do tratamento $(70,72)$. Um estudo com 30 pacientes mostrou que o grupo teste $(n=15)$, que recebeu $400 \mathrm{mg}$ de HQ uma vez ao dia por 5 dias, apresentou 86,7\% dos indivíduos com teste nasofarígeo negativo para COVID-19 após 7 dias. No entanto, não houve diferenças significativas em relação ao grupo controle $(\mathrm{n}=15)$, que apresentou 93,3\% dos indivíduos curados (73). Nesse estudo, todos os pacientes apresentavam sintomas comuns e em ambos os grupos havia até 6 pacientes portadores de comorbidades (73).

Até a data desta pesquisa, 81 ensaios clínicos (EC) intervencionistas, distribuídos entre as fases I e IV, estão analisando seus benefícios em monoterapia (20 EC) e com outros fármacos adjuvantes (39 EC), principalmente com a azitromicina (19EC) e fármacos antivirais (7 EC), além de sua avaliação como agente profilático (22 EC),todos disponíveis no site ClinicalTrials.GOV.

A HCQ é administrada como sulfato e possui um nível menor de acumulação tecidual, estando menos associada a efeitos adversos quando comparada com CLQ (61). As recomendações atuais do Ministério da Saúde do Brasil para o uso de
HCQ na terapia de pacientes com COVID-19 é de $800 \mathrm{mg}$ no primeiro dia, seguido de $400 \mathrm{mg} /$ dia durante 5 dias (67). Esses valores já haviam sido recomendados por um estudo que simulou as concentrações pulmonares de HCQ, a fim de avaliar concentrações eficientes e que não atingissem níveis não-seguros (63).

Embora seja relatado um bom perfil de segurança deste fármaco, a HCQ pode ter como complicação mais grave o desenvolvimento de retinopatia, principalmente se administrada cronicamente (períodos $>5$ anos) e em doses altas (74). Ademais, pode ocorrer predisposição a arritmias em pacientes graves e com fatores de risco, um efeito que pode ser aprimorado pelo uso simultâneo de AZ, assim como o aumento do intervalo QT (61).

\section{Ivermectina}

A ivermectina (IVM) é um antiparasitário usado na medicina humana e veterinária. Na saúde humana, possui forte ação contra endoparasitas e ectoparasitas, sendo um agente importante no tratamento de patologias como oncocersose, filariose linfática e ascaridíase (75). Ao ser administrada, essa substância é amplamente distribuída pelos tecidos corporais, metabolizada pelas enzimas do CIP450 e excretada pelas fezes (76).

Há alguns anos foi relatado que a IVM possui atividade in vitro contra alguns flavivírus, incluindo DENV e HIV-1 $(77,78)$. Com bases nesses resultados, foi demonstrado que IVM também possui ação anti-SARS-CoV-2 in vitro $(5 \mu \mathrm{M}$ em cultura de células Vero/hSLAM infectadas com SARS-CoV-2), havendo perda de quase todo material viral em 48h (79). No entanto, até o momento, nenhum estudo clínico demonstrou se essa eficácia é alcançada in vivo e qual a segurança em utilizá-la, não existindo, portanto, recomendações estabelecidas quanto ao seu uso no tratamento de pacientes infectados com COVID-19.

Até a data da presente pesquisa, quatro ensaios clínicos (EC) intervencionistas, distribuídos entre as fases I e III, estão analisando seu uso associado a outros fármacos: bicalutamida (80), nitazoxanida (81) e uma associação com HCQ/AZ (82). 


\section{Nitazoxanida}

A nitazoxanida é anti-helmíntico usado principalmente no tratamento de infecções intestinais, como amebíase e giardíase. Sua administração é feita por via oral, sendo rapidamente absorvida pelo trato gastrointestinal, onde sofre hidrólise e forma o seu metabólito ativo, a tizoxanidale, o qual já foi descrito como um bloqueador in vitro de cepas de influenza A e B, vírus da hepatite $\mathrm{B}$ e $\mathrm{C}$, norovírus, entre outros (83).

Recentemente, foi relatada sua ação anti-SAR$\mathrm{S}-\mathrm{CoV}-2$ in vitro com $\mathrm{EC}_{50}=2,12 \mu \mathrm{M}(6)$. No entanto, ainda não foram divulgados outros estudos que relatassem sua eficácia no uso em pacientes com COVID-19. Ensaios clínicos com a nitazoxanida como uma opção terapêutica estão ativos e disponíveis no site ClinicalTrials.GOV, principalmente como fármaco adjuvante da HCQ e IVM, embora ainda sem dados disponíveis $(84,85)$.

\section{CONCLUSÃO}

Todos os antivirais testados contra o SARS-CoV-2 são fármacos utilizados para o tratamento de doenças causadas por outros vírus, como o HIV, o vírus do ebola e da Influenza. Infelizmente, ainda não é possível definir um fármaco antiviral que seja uma opção terapêutica resolutiva contra a COVID-19. A cloroquina e a hidroxicloroquina, que já demonstraram ação na melhora clínica e na diminuição da carga viral em pacientes diagnosticados com COVID-19, e o Remdesivir, que já está em fase III de estudos, são os fármacoss em situação mais avançada como potenciais agentes terapêuticos para o tratamento da COVID-19. Os esforços que estão sendo aplicados nos estudos clínicos atual irão contribuir na identificação de dados relacionados à eficácia, segurança e dosagens desses fármacos para que promovam impacto significativo na redução da morbimortalidade dos pacientes.

\section{REFERÊNCIAS}

1. Zhu N, Zhang D, Wang W, Li X, Yang B, Song J, Zhao X, Huang B, Shi W, Lu R, Niu P, Zhan F, Ma X, Wang D, Xu W, Wu G, Gao GF, Tan W; China Novel Coronavirus Investigating and Research Team. A Novel Coronavirus from Patients with Pneumonia in China, 2019. N Engl J Med. 2020;382(8):727-733. DOI: 10.1056/ NEJMoa2001017.

2. WHO. World Health Organization Coronavirus Disease (COVID-19) Dashboard. [cited 2020 June 12]. Available from:https://covid19.who.int/

3. Zhou P, Yang XL, Wang XG, Hu B, Zhang L, Zhang W, Si HR, Zhu Y, Li B, Huang CL, Chen HD, Chen J, Luo Y, Guo H, Jiang RD, Liu MQ, Chen Y, Shen XR, Wang X, Zheng XS, Zhao K, Chen QJ, Deng F, Liu LL, Yan B, Zhan FX, Wang YY, Xiao GF, Shi ZL. A pneumonia outbreak associated with a new coronavirus of probable bat origin. Nature.2020;579(7798):270-273. DOI: 10.1038/s41586-020-2012-7.

4. Huang C, Wang Y, Li X, Ren L, Zhao J, Hu Y, Zhang L, Fan G, Xu J, Gu X, Cheng Z, Yu T, Xia J, Wei Y, Wu W, Xie X, Yin W, Li H, Liu M, Xiao Y, Gao H, Guo L, Xie J, Wang G, Jiang R, Gao Z, Jin Q, Wang J, Cao B. Clinical features of patients infected with 2019 novel coronavirus in Wuhan, China. Lancet. 2020;395(10223):497-506. DOI: 10.1016/S0140-6736(20)30183-5.

5. Scavone C, Brusco S, Bertini M, Sportiello L, Rafaniello C, Zoccoli A, Berrino L, Racagni G, Rossi F, Capuano
A. Current pharmacological treatments for COVID-19: What's next? Br J Pharmacol. 2020;10.1111/bph.15072. DOI: 10.1111/bph.15072.

6. Wang M, Cao R, Zhang L, Yang X, Liu J, Xu M, Shi Z, Hu Z, Zhong W, Xiao G. Remdesivir and chloroquine effectively inhibit the recently emerged novel coronavirus (2019-nCoV) in vitro. Cell Res. 2020;30(3):269-271. DOI: 10.1038/s41422-020-0282-0.

7. Reina J. Remdesivir, the antiviral hope against SARSCoV-2. Rev Esp Quimeter. 2020;33(3):176-179. DOI: 10.37201/req/028.2020.

8. ClinicalTrials.gov [Internet]. Bethesda (MD): National Library of Medicine (US). 2000 Feb 29. Identifier NCT04292899, Study to Evaluate the Safety and Antiviral Activity of Remdesivir (GS-5734TM) in Participants With Severe Coronavirus Disease (COVID-19); 2020 Mar 3 [cited 2020 May 5]; [about 4 screens]. Available from: https://clinicaltrials.gov/ct2/show/NCT04292899

9. WHO. World Health Organization. WHO R\&D Blueprint - Informal consultation on prioritization of candidate therapeutic agents for use in novel coronavirus 2019 infection.2020.

10. Norrie JD. Remdesivir for COVID-19: challenges of underpowered studies. Lancet. 2020;395(10236):15251527. DOI: 10.1016/S0140-6736(20)31023-0. 
11. Mehta N, Mazer-Amirshahi M, Alkindi N, Pourmand A. Pharmacotherapy in COVID-19; A narrative review for emergency providers. Am J Emerg Med. 2020:S07356757(20)30263-1. DOI: 10.1016/j.ajem.2020.04.035.

12. Furuta Y, Gowen BB, Takahashi K, Shiraki K, Smee DF, Barnard DL. Favipiravir (T-705), a novel viral RNA polymerase inhibitor. Antiviral Res. 2013;100(2):446-454. DOI: 10.1016/j.antiviral.2013.09.015.

13. Gatherer D. The 2014 Ebola virus disease outbreak in West Africa. J Gen Virol. 2014;95(Pt 8):1619-1624. DOI:10.1099/vir.0.067199-0

14. Furuta Y, Komeno T, Nakamura T. Favipiravir (T-705), a broad spectrum inhibitor of viral RNA polymerase. Proc Jpn Acad Ser B Phys Biol Sci. 2017;93(7):449-463. DOI:10.2183/pjab.93.027

15. Li G, De Clercq E. Therapeutic options for the 2019 novel coronavirus (2019-nCoV). Nat Rev Drug Discov. 2020;19(3):149-150. DOI: 10.1038/d41573-020-00016-0.

16. Shiraki K, Daikoku T. Favipiravir, an anti-influenza drug against life-threatening RNA virus infections. PharmacolTher. 2020 May;209:107512. doi: 10.1016/ j.pharmthera.2020.107512.

17. Delang L, Abdelnabi R, Neyts J. Favipiravir as a potential countermeasure against neglected and emerging RNA viruses. Antiviral Res. 2018;153:85-94. DOI: 10.1016/ j.antiviral.2018.03.003.

18. ClinicalTrials.gov [Internet], Bethesda (MD): National Library of Medicine (US). 2000 Feb 29. Identifier NCT04319900, Clinical Trial of Favipiravir Tablets Combine With Chloroquine Phosphate in the Treatment of Novel Coronavirus Pneumonia; 2020 Mar 24 [cited 2020 May 8]; [about 4 screens]. Available from: https:// clinicaltrials.gov/ct2/show/NCT04319900

19. Costanzo M, De Giglio MAR, Roviello GN. SARSCoV-2: Recent Reports on Antiviral Therapies Based on Lopinavir/Ritonavir, Darunavir/Umifenovir, Hydroxychloroquine, Remdesivir, Favipiravir and Other Drugs for the Treatment of the New Coronavirus [published online ahead of print, 2020 Apr 16]. Curr Med Chem. 2020 ;10.2174/0929867327666200416131117. DOI: 10.2174/ 0929867327666200416131117

20. Choy KT, Wong AY, Kaewpreedee P, Sia SF, Chen D, Hui KPY, Chu DKW, Chan MCW, Cheung PP, Huang X, Peiris M, Yen HL. Remdesivir, lopinavir, emetine, and homoharringtonine inhibit SARS-CoV-2 replication in vitro. Antiviral Res. 2020;178:104786. DOI: 10.1016/ j.antiviral.2020.104786.

21. Chinello P, Petrosillo N, Pittalis S, Biava G, Ippolito G, Nicastri E. INMI Ebola Team. QTc interval prolongation during favipiravir therapy in an Ebolavirus-infected patient. PLoSNegl Trop Dis. 2017;11(12):e0006034. DOI: 10.1371/journal.pntd.0006034.
22. Martinez MA. Compounds with Therapeutic Potential against Novel Respiratory 2019 Coronavirus. Antimicrob Agents Chemother. 2020;64(5):e00399-20. DOI: 10.1128/AAC.00399-20.

23. Salvi R, Patankar P. Emerging pharmacotherapies for COVID-19. Biomed Pharmacother. 2020;128:110267. DOI: 10.1016/j.biopha.2020.110267.

24. Zhong H, Wang Y, Zhang ZL, Liu YX, Le KJ, Cui M, Yu YT, Gu ZC, Gao Y, Lin HW. Efficacy and safety of current therapeutic options for COVID-19 - lessons to be learnt from SARS and MERS epidemic: A systematic review and meta-analysis. Pharmacol Res. 2020:104872. DOI: 10.1016/j.phrs.2020.104872.

25. Chu CM, Cheng VC, Hung IF, Wong MM, Chan KH, Chan KS, Kao RY, Poon LL, Wong CL, Guan Y, Peiris JS, Yuen KY; HKU/UCH SARS Study Group. Role of lopinavir/ritonavir in the treatment of SARS: initial virological and clinical findings. Thorax. 2004;59(3):252-6. DOI: 10.1136/thorax.2003.012658.

26. Kumar S, Zhi K, Mukherji A, Gerth K. Repurposing Antiviral Protease Inhibitors Using Extracellular Vesicles for Potential Therapy of COVID-19. Viruses. 2020;12(5). pii: E486. DOI: 10.3390/v12050486.

27. McKee DL, Sternberg A, Stange U, Laufer S, Naujokat C. Candidate drugs against SARS-CoV-2 and COVID-19. Pharmacol Res. 2020;157:104859. DOI: 10.1016/j.phrs.2020.104859.

28. ClinicalTrials.gov [Internet]. Bethesda (MD): National Library of Medicine (US). 2000 Feb 29. Identifier NCT04321174, COVID-19 Ring-based Prevention Trial With Lopinavir/Ritonavir (CORIPREV-LR); 2020 Mar 25 [cited 2020 May 9]; [about 4 screens]. Available from:https://clinicaltrials.gov/ct2/show/NCT04321174

29. ClinicalTrials.gov [Internet]. Bethesda (MD): National Library of Medicine (US). 2000 Feb 29. Identifier NCT04252885, The Efficacy of Lopinavir Plus Ritonavir and Arbidol Against Novel Coronavirus Infection (ELACOI); 2020 Feb 5 [cited 2020 May 11]; [about 4 screens]. Available from:https://clinicaltrials.gov/ct2/ show/NCT04252885

30. Cao B, Wang Y, Wen D, Liu W, Wang J, Fan G, Ruan L, Song B, Cai Y, Wei M, Li X, Xia J, Chen N, Xiang J, Yu T, Bai T, Xie X, Zhang L, Li C, Yuan Y, Chen H, Li H, Huang H, Tu S, Gong F, Liu Y, Wei Y, Dong C, Zhou F, Gu X, Xu J, Liu Z, Zhang Y, Li H, Shang L, Wang K, Li K, Zhou X, Dong X, Qu Z, Lu S, Hu X, Ruan S, Luo S, Wu J, Peng L, Cheng F, Pan L, Zou J, Jia C, Wang J, Liu X, Wang S, Wu X, Ge Q, He J, Zhan H, Qiu F, Guo L, Huang C, Jaki T, Hayden FG, Horby PW, Zhang D, Wang C. A Trial of Lopinavir-Ritonavir in Adults Hospitalized with Severe Covid-19. N Engl J Med. 2020;382(19):1787-1799. DOI: 10.1056/NEJMoa2001282. 
31. Hung IF, Lung KC, Tso EY, Liu R, Chung TW, Chu MY, Ng YY, Lo J, Chan J, Tam AR, Shum HP, Chan V, Wu AK, Sin KM, Leung WS, Law WL, Lung DC, Sin S, Yeung P, Yip CC, Zhang RR, Fung AY, Yan EY, Leung KH, Ip JD, Chu AW, Chan WM, Ng AC, Lee R, Fung K, Yeung A, Wu TC, Chan JW, Yan WW, Chan WM, Chan JF, Lie AK, Tsang OT, Cheng VC, Que TL, Lau CS, Chan KH, To KK, Yuen KY. Triple combination of interferon beta-1b, lopinavir-ritonavir, and ribavirin in the treatment of patients admitted to hospital with COVID-19: an open-label, randomised, phase 2 trial. Lancet. 2020;395(10238):16951704. DOI: $10.1016 / \mathrm{S} 0140-6736(20) 31042-4$.

32. Ye XT, Luo YL, Xia SC, Sun QF, Ding JG, Zhou Y, Chen W, Wang XF, Zhang WW, Du WJ, Ruan ZW, Hong L. Clinical efficacy of lopinavir/ritonavir in the treatment of Coronavirus disease 2019. Eur Rev Med Pharmacol Sci. 2020 Mar;24(6):3390-3396. DOI: 10.26355/eurrev _202003_20706.

33. Naksuk N, Lazar S, Peeraphatdit TB. Cardiac safety of off-label COVID-19 drug therapy: a review and proposed monitoring protocol. Eur Heart J Acute Cardiovasc Care. 2020:2048872620922784. DOI: $10.1177 / 2048872620922784$.

34. Agrawal R, Rewatkar PV, Kokil GR, Verma A, Kalra A. Oseltamivir: A first line defense against swine flu. Med Chem. 2010;6(4):247-51. DOI: $10.2174 / 1573406411006040247$.

35. Lampejo T. Influenza and antiviral resistance: an overview [published online ahead of print, 2020 Feb 13]. Eur J Clin Microbiol Infect Dis. 2020;1-8. DOI: 10.1007/ s10096-020-03840-9

36. Chen $\mathrm{Y}, \mathrm{Ke} \mathrm{M}, \mathrm{Xu}$ J, Lin C. Simulation of the Pharmacokinetics of Oseltamivir and Its Active Metabolite in Normal Populations and Patients with Hepatic Cirrhosis Using Physiologically Based Pharmacokinetic Modeling. AAPS PharmSciTech. 2020;21(3):98. DOI: 10.1208/s12249-020-1638-y.

37. Ayerbe L, Risco C, Ayis S. The association between treatment with heparin and survival in patients with Covid-19. J Thromb Thrombolysis. 2020;1:1-4. DOI: 10.1007/s11239-020-02162-z.

38. ClinicalTrials.gov [Internet]. Bethesda (MD): National Library of Medicine (US). 2000 Feb 29. Identifier NCT04303299, Various Combination of Protease Inhibitors, Oseltamivir, Favipiravir, and Hydroxychloroquine for Treatment of COVID-19: A Randomized Control Trial (THDMS-COVID-19); 2020 Mar 11 [cited 2020 May 12]; [about 4 screens]. Available from:https://clinicaltrials. gov/ct2/show/results/NCT04303299?view=results

39. Wang D, Hu B, Hu C, Zhu F, Liu X, Zhang J, Wang B, Xiang H, Cheng Z, Xiong Y, Zhao Y, Li Y, Wang X, Peng Z. Clinical Characteristics of 138 Hospitalized Patients With 2019 Novel Coronavirus-Infected Pneumonia in Wuhan, China. JAMA. 2020;323(11):1061-1069. DOI: 10.1001/jama.2020.1585.
40. Jefferson T, Jones M, Doshi P, Spencer EA, Onakpoya I, Heneghan CJ. Oseltamivir for influenza in adults and children: systematic review of clinical study reports and summary of regulatory comments. Version 2. BMJ. 2014;348:g2545. DOI: 10.1136/bmj.g2545.

41. Gao X, Rosales A, Karttunen H, Bommana GM, Tandoh B, Yi Z, Habib Z, D'Agati V, Zhang W, Ross MJ. The HIV protease inhibitor darunavir prevents kidney injury via HIV-independent mechanisms. Sci Rep. 2019;9(1):15857. DOI: 10.1038/s41598-01952278-3.

42. Colbers A, Greupink R, Litjens C, Burger D, Russel FG. Physiologically Based Modelling of Darunavir/Ritonavir Pharmacokinetics During Pregnancy. Clin Pharmacokinet. 2016;55(3):381-396. DOI: 10.1007/s40262-0150325-8.

43. Zakharova MY, Kuznetsova AA, Kaliberda EN, Dronina MA, Kolesnikov AV, Kozyr AV, Smirnov IV, Rumsh LD, Fedorova OS, Knorre DG, Gabibov AG, Kuznetsov NA. Evolution of inhibitor-resistant natural mutant forms of HIV-1 protease probed by pre-steady state kinetic analysis. Biochimie. 2017;142:125-134. DOI: 10.1016/ j.biochi.2017.08.014.

44. Pant S, Singh M, Ravichandiran V, Murty USN, Srivastava HK. Peptide-like and small-molecule inhibitors against Covid-19. J Biomol Struct Dyn. 2020;6:1-10. DOI: 10.1080/07391102.2020.1757510.

45. Alpern JD, Gertner E. Off-Label Therapies for COVID-19-Are We All In This Together? Clin Pharmacol Ther. 2020; 10.1002/cpt.1862. DOI: 10.1002/cpt.1862.

46. Dong L, Hu S, Gao J. Discovering drugs to treat coronavirus disease 2019 (COVID-19). Drug Discov Ther. 2020;14(1):58-60. DOI: 10.5582/ddt.2020.01012.

47. ClinicalTrials.gov [Internet]. Bethesda (MD): National Library of Medicine (US). 2000 Feb 20. Identifier NCT04252274, Efficacy and Safety of Darunavir and Cobicistat for Treatment of COVID-19 (DCCOVID-19); 2020 Feb 5 [cited 2020 May 13]; [about 4 screens]. Available from:https://clinicaltrials.gov/ct2/ show/NCT04252274

48. Putcharoen O, Do T, Avihingsanon A, Ruxrungtham K. Rationale and clinical utility of the darunavir-cobicistat combination in the treatment of HIV/AIDS. Drug Des Devel Ther. 2015;9:5763-5769. DOI: 10.2147/DDDT. S63989.

49. Deeks ED. Darunavir: a review of its use in the management of HIV-1 infection. Drugs. 2014;74(1):99-125. DOI: 10.1007/s40265-013-0159-3.

50. Leneva IA, Russell RJ, Boriskin YS, Hay AJ. Characteristics of arbidol-resistant mutants of influenza virus: implications for the mechanism of anti-influenza action of arbidol. Antiviral Res. 2009;81(2):132-140. DOI: 10.1016/j.antiviral.2008.10.009. 
51. Rashad AA, Mahalingam S, Keller PA. Chikungunya virus: emerging targets and new opportunities for medicinal chemistry. J Med Chem. 2014;57(4):1147-66. DOI: 10.1021/jm400460d.

52. Boriskin YS, Pécheur EI, Polyak SJ. Arbidol: a broad-spectrum antiviral that inhibits acute and chronic HCV infection. Virol J. 2006;3:56. DOI: 10.1186/1743422X-3-56.

53. Blaising J, Polyak SJ, Pécheur EI. Arbidol as a broad-spectrum antiviral: an update. Antiviral Res. 2014;107:84-94. DOI: 10.1016/j.antiviral.2014.04.006. Epub 2014 Apr 24.

54. Zhu Z, Lu Z, Xu T, Chen C, Yang G, Zha T, Lu J, Xue Y. Arbidol monotherapy is superior to lopinavir/ ritonavir in treating COVID-19. J Infect. 2020;S01634453(20)30188-2. DOI: 10.1016/j.jinf.2020.03.060.

55. Deng L, Li C, Zeng Q, Liu X, Li X, Zhang H, Hong Z, $\mathrm{Xia} \mathrm{J}$. Arbidol combined with $\mathrm{LPV} / \mathrm{r}$ versus $\mathrm{LPV} / \mathrm{r}$ alone against Corona Virus Disease 2019: A retrospective cohort study. J Infect. 2020;S0163-4453(20):3011330114. DOI: 10.1016/j.jinf.2020.03.002.

56. Pshenichnaya NY, Bulgakova VA, Lvov NI, Poromov AA, Selkova EP, Grekova AI, Shestakova IV, Maleev VV, Leneva IA. Clinical efficacy of umifenovir in influenza and ARVI (study ARBITR).TerArkh. 2019;91(3):56-63. DOI: 10.26442/00403660.2019.03.000127.

57. Mackenzie AH. Pharmacologic actions of 4-aminoquinoline compounds. Am J Med. 1983;75(1A):5-10. DOI: 10.1016/0002-9343(83)91264-0

58. Ducharme J, Farinotti R. Clinical pharmacokinetics and metabolism of chloroquine. Focus on recent advancements. Clin Pharmacokinet. 1996;31(4):257-274. DOI: 10.2165/00003088-199631040-00003

59. Savarino A, Boelaert JR, Cassone A, Majori G, Cauda R. Effects of chloroquine on 538 viral infections: an old drug against today's diseases?. Lancet Infect Dis. 2003;3(11):722-727. DOI: 10.1016/s1473-3099(03) 00806-5

60. Krogstad DJ, Schlesinger PH. Acid-vesicle function, intracellular pathogens, and the action of chloroquine against Plasmodium falciparum. N Engl J Med. 1987;317(9):542-549. DOI: 10.1056/NEJM198708273 170905

61. Schrezenmeier E, Dörner T. Mechanisms of action of hydroxychloroquine and chloroquine: implications for rheumatology. Nat Rev Rheumatol. 2020;16(3):155-166. DOI: $10.1038 / \mathrm{s} 41584-020-0372-\mathrm{X}$

62. Vincent MJ, Bergeron E, Benjannet S, Erickson BR, Rollin PE, Ksiazek TG, Seidah NB, Nichol ST. Chloroquine is a potent inhibitor of SARS coronavirus infection and spread. Virol J. 2005;2:69. DOI: 10.1186/1743$422 \mathrm{X}-2-69$
63. Yao X, Ye F, Zhang M, Cui C, Huang B, Niu P, Liu X, Zhao L, Dong E, Song C, Zhan S, Lu R, Li H, Tan W, Liu D. In Vitro Antiviral Activity and Projection of Optimized Dosing Design of Hydroxychloroquine for the Treatment of Severe Acute Respiratory Syndrome Coronavirus 2 (SARS-CoV-2) [published online ahead of print, 2020 Mar 9]. Clin Infect Dis. 2020; ciaa237. DOI: 10.1093/cid/ciaa237

64. Fantini J, Di Scala C, Chahinian H, Yahi N. Structural and molecular modelling studies reveal a new mechanism of action of chloroquine and hydroxychloroquine against SARS-CoV-2 infection. Int J Antimicrob Agents. 2020;55(5):105960. DOI: 10.1016/j.ijantimicag. 2020.105960

65. Liu J, Cao R, Xu M, Wang X, Zhang H, Hu H, Li Y, $\mathrm{Hu} \mathrm{Z}$, Zhong W, Wang M. Hydroxychloroquine, a less toxic derivative of chloroquine, is effective in inhibiting SARS-CoV-2 infection in vitro. Cell Discov. 2020;6:16. DOI: $10.1038 / \mathrm{s} 41421-020-0156-0$

66. Gao J, Tian Z, Yang X. Breakthrough: Chloroquine phosphate has shown apparent efficacy in treatment of COVID-19 associated pneumonia in clinical studies. Biosci Trends. 2020;14(1):72-73. DOI: 10.5582/bst. 2020.01047

67. BRASIL. Ministério da Saúde. Diretrizes para diagnóstico e tratamento da COVID-19. $3^{\mathrm{a}}$ ed.Brasília (DF); 2020.

68. Juurlink DN. Safety considerations with chloroquine, hydroxychloroquine and azithromycin in the management of SARS-CoV-2 infection. CMAJ. 2020;192(17): E450-E453. DOI: $10.1503 /$ cmaj.200528

69. Devaux CA, Rolain JM, Colson P, Raoult D. New insights on the antiviral effects of chloroquine against coronavirus: what to expect for COVID-19?. Int J Antimicrob Agents. 2020;55(5):105938. DOI: 10.1016/j. ijantimicag.2020.105938

70. Gautret P, Lagier JC, Parola P, Hoang VT, Meddeb L, Mailhe M, Doudier B, Courjon J, Giordanengo V, Vieira VE, Dupont HT, Honoré S, Colson P, Chabrière E, La Scola B, Rolain JM, Brouqui P, Raoult D. Hydroxychloroquine and azithromycin as a treatment of COVID-19: results of an open-label non-randomized clinical trial [published online ahead of print, 2020 Mar 20]. Int J Antimicrob Agents. 2020;105949. DOI: 10.1016/j. ijantimicag.2020.105949.

71. Gautret P, Lagier JC, Parola P, Hoang VT, Meddeb L, Sevestre J, Mailhe M, Doudier B, Aubry C, Amrane S, Seng P, Hocquart M, Eldin C, Finance J, Vieira VE, Tissot-Dupont HT, Honoré S, Stein A, Million M, Colson P, La Scola B, Veit V, Jacquier A, Deharo JC, Drancourt M, Fournier PE, Rolain JM, Brouqui P, Raoult D. Clinical and microbiological effect of a combination of hydroxychloroquine and azithromycin in 80 COVID-19 patients with at least a six-day follow up: A pilot observational study. Travel Med Infect Dis. 2020;34:101663. DOI: 10.1016/j.tmaid.2020.101663 
72. Molina JM, Delaugerre C, Le Goff J, Mela-Lima B, Ponscarme D, Goldwirt L, Castro N. No evidence of rapid antiviral clearance or clinical benefit with the combination of hydroxychloroquine and azithromycin in patients with severe COVID-19 infection. Med Mal Infect. 2020;50(4):384. DOI: 10.1016/j.medmal.2020.03.006

73. Chen J, Liu D, Liu L, Liu P, Xu Q, Xia L, Ling Y, Huang D, Song S, Zhang D, Qian Z, Li T, Shen Y, Lu H. [A pilot study of hydroxychloroquine in treatment of patients with moderate COVID-19]. J Zhejiang Univ (Med Sci). 2020;1: 0-0. DOI: 10.3785/j.issn.1008-9292.2020.03.03

74. Jorge A, Ung C, Young LH, Melles RB, Choi HK. Hydroxychloroquine retinopathy - implications of research advances for rheumatology care. Nat Rev Rheumatol. 2018;14(12):693-703. DOI: 10.1038/s41584-018-0111-8

75. Laing R, Gillan V, Devaney E. Ivermectin - Old Drug, New Tricks? Trends Parasitol. 2017;33(6):463-472. DOI: 10.1016/j.pt.2017.02.004

76. González Canga A, Sahagún Prieto AM, Diez Liébana MJ, Fernández Martínez N, Sierra Vega M, García Vieitez JJ. The pharmacokinetics and interactions of ivermectin in humans--a mini-review. AAPS J. 2008;10(1):42-46. DOI: $10.1208 / \mathrm{s} 12248-007-9000-9$

77. Mastrangelo E, Pezzullo M, De Burghgraeve T, Kaptein S, Pastorino B, Dallmeier K, de Lamballerie X, Neyts J, Hanson AM, Frick DN, Bolognesi M, Milani M. Ivermectin is a potent inhibitor of flavivirus replication specifically targeting NS3 helicase activity: new prospects for an old drug. J Antimicrob Chemother. 2012;67(8):1884-1894. DOI: 10.1093/jac/dks 147

78. Wagstaff KM, Sivakumaran H, Heaton SM, Harrich $\mathrm{D}$, Jans DA. Ivermectin is a specific inhibitor of importin $\alpha / \beta$-mediated nuclear import able to inhibit replication of HIV-1 and dengue virus. Biochem J. 2012; 443(3):851-856. DOI: 10.1042/BJ20120150

79. Caly L, Druce JD, Catton MG, Jans DA, Wagstaff KM. The FDA-approved drug ivermectin inhibits the replication of SARS-CoV-2 in vitro [published online ahead of print, 2020 Apr 3]. Antiviral Res. 2020;178:104787. DOI: $10.1016 /$ j.antiviral.2020.104787
80. ClinicalTrials.gov [Internet]. Bethesda (MD): National Library of Medicine (US). 2000 Feb 29. Identifier NCT04374279, Trial to Promote Recovery From COVID-19 With Ivermectin or Endocrine Therapy (RECOVER); 2020 May 5 [cited 2020 May 20]; [about 4 screens]. Available from: https://clinicaltrials.gov/ct2/ show/study/NCT04374279

81. ClinicalTrials.gov [Internet]. Bethesda (MD): National Library of Medicine (US). 2000 Feb 29. Identifier NCT04360356, Ivermectin and Nitazoxanide Combination Therapy for COVID-19; 2020 April 24 [cited 2020 May 20]; [about 4 screens]. Available from: https:// clinicaltrials.gov/ct2/show/NCT04360356

82. ClinicalTrials.gov [Internet]. Bethesda (MD): National Library of Medicine (US). 2000 Feb 29. Identifier NCT04374019, Novel Agents for Treatment of Highrisk COVID-19 Positive Patients; 2020 May 5 [cited 2020 May 20]; [about 4 screens]. Available from: https:// clinicaltrials.gov/ct2/show/NCT04374019

83. Rossignol JF. Nitazoxanide: a first-in-class broad-spectrum antiviral agent. Antiviral Res. 2014;110:94-103. DOI: 10.1016/j.antiviral.2014.07.014

84. ClinicalTrials.gov [Internet]. Bethesda (MD): National Library of Medicine (US). 2000 Feb 29. Identifier NCT04343248, Trial to Evaluate the Efficacy and Safety of Nitazoxanide (NTZ) for Post-Exposure Prophylaxis of COVID-19 and Other Viral Respiratory Illnesses in Elderly Residents of Long-Term Care Facilities (LTCF); 2020 April 13 [cited 2020 May 27]; [about 4 screens]. Available from: https://clinicaltrials.gov/ct2/show/ NCT04343248

85. ClinicalTrials.gov [Internet]. Bethesda (MD): National Library of Medicine (US). 2000 Feb 29. Identifier NCT04348409, Efficacy and Safety of Nitazoxanide for the Treatment of Hospitalized Patients With Moderate COVID-19; 2020 April 16 [cited 2020 May 27]; [about 4 screens]. Available from: https://clinicaltrials.gov/ct2/ show/NCT0434840 